\title{
The role of hydrophobicity in the cold denaturation of proteins under high pressure: A study on apomyoglobin
}

\author{
Cite as: J. Chem. Phys. 150, 075102 (2019); doi: 10.1063/1.5080942 \\ Submitted: 12 November 2018 - Accepted: 31 January 2019 • \\ Published Online: 21 February 2019
}

Yanis R. Espinosa, ${ }^{1, a)}$ (D) Ernesto R. Caffarena, ${ }^{2}$ (D) and J. Raúl Grigera ${ }^{3}$ (D)

\begin{abstract}
AFFILIATIONS
'Instituto de Física de Líquidos y Sistemas Biológicos (CONICET-UNLP), Calle 59 Nro 789, B1900BTE La Plata, Argentina

${ }^{2}$ Programa de Computação Científica (PROCC), Fundação Oswaldo Cruz, Manguinhos, CEP 21040-360 Rio de Janeiro, Brazil

${ }^{3}$ CEQUINOR, Universidad de La Plata and CONICET, 47 y 115, B1900 La Plata, Argentina
\end{abstract}

a) Author to whom correspondence should be addressed: respinosa@iflysib.unlp.edu.ar

\begin{abstract}
An exciting debate arises when microscopic mechanisms involved in the denaturation of proteins at high pressures are explained. In particular, the issue emerges when the hydrophobic effect is invoked, given that hydrophobicity cannot elucidate by itself the volume changes measured during protein unfolding. In this work, we study by the use of molecular dynamics simulations and essential dynamics analysis the relation between the solvation dynamics, volume, and water structure when apomyoglobin is subjected to a hydrostatic pressure regime. Accordingly, the mechanism of cold denaturation of proteins under high-pressure can be related to the disruption of the hydrogen-bond network of water favoring the coexistence of two states, low-density and high-density water, which directly implies in the formation of a molten globule once the threshold of $200 \mathrm{MPa}$ has been overcome.
\end{abstract}

Published under license by AIP Publishing. https://doi.org/1 0.1 063/1.5080942

\section{INTRODUCTION}

Hydrostatic pressure is extensively used for studying protein folding ${ }^{1-3}$ and can be applied as a perturbative method to destabilize globular proteins. ${ }^{3}$ On these, the pressure induces changes including small conformational effects, compressibility changes, ${ }^{7}$ and local effects on regions containing internal cavities. ${ }^{3}$

According to the protein volume theorem proposed by Akasaka "the partial molar volume of a protein decreases in parallel with the loss of its conformational order."4 In other words, a protein in solution is a dynamic mixture of various conformational sub-states that varies the partial molar volume and Gibbs energy. Thus, with increasing pressure, the conformational order decreases concomitantly with its partial molar volume (negative $\Delta \mathrm{V}$ ) as a result of small positive Gibbs energy increment $(\Delta G>0))^{5,6}$ Therefore, protein folding/unfolding is controlled by Le Chatelier's principle. ${ }^{7}$
Moreover, the structural backgrounds of unfolding are characterized for (i) an increased hydration of the polypeptide chain, ${ }^{5}$ favoring water infiltration into the protein ${ }^{7}$ along with the disassembly of protein cavities, ${ }^{3,8}$ (ii) changes in the solvent density through electrostriction of the exposed charged residues, ${ }^{9}$ and (iii) modification on the water structure. ${ }^{10,11}$

Likewise, changes in the solvent-exposed surface area have also been used to explain the effects of pressure on proteins. $^{12}$ It was already observed that although the $\Delta \mathrm{V}$ associated with the protein cannot correlate directly with the change in the surface area upon unfolding, ${ }^{13}$ its volume changes due to the hydration of those atoms remaining inaccessible to the solvent in the native state but become exposed upon unfolding. ${ }^{7}$ However, there are those who would argue that the denaturation by pressure should focus on the free energy of water transfer into the hydrophobic core of the protein rather than a transfer of non-polar solutes into water. ${ }^{14}$ 
However, it is well known that the high pressure prompts the protein to visit a population of conformational states that are more solvated than the native state. ${ }^{1}$ At the same time, the water structure is highly influenced by pressure, generating structural anomalies in the liquid phase, ${ }^{15}$ where the first coordination shell around the solute is destabilized, the hydrogen bonds (HBs) are redistributed, and the water density is modified. ${ }^{10,16-18}$ Thus, these changes at high pressures can be understood as the water structural transformation from an open tetrahedral structure (Low-Density Water, LDW) into a compact hexagonal one (High-Density Water, HDW), ${ }^{17}$ which is evident $\sim 200 \mathrm{MPa}$ at room temperature, ${ }^{18}$ allowing water penetration into a hydrophobic core. ${ }^{19}$

So, what is the role of hydrophobicity and the volume change in cold denaturation of proteins under high pressure? How is the change in the water structure associated with the increased hydration of the polypeptide chain?

To try and answer these questions, in this work, we carried out a study of the effects of pressure on apomyoglobin (a globular protein widely studied theoretically ${ }^{12,20,21}$ and experimentally, 22-24 and with which our group has studied the behavior of the hydrophobic effect under pressure ${ }^{12}$ ) to analyze the relation between the solvation dynamics, volume, and water structure employing computational techniques for understanding this process.

\section{COMPUTATIONAL METHODS}

Molecular dynamics (MD) simulations were performed using the Gromacs 4.6.7 package. ${ }^{25,26}$ To account for intermolecular interactions, the Gromos $43 \mathrm{a}^{27}$ force field was employed. Initial atomic coordinates were taken from the crystal structure of sperm whale myoglobin deposited in the Protein Data Bank (PDB code VXD $^{28}$ ). The apomyoglobin topology was generated taking into account standard $\mathrm{pH} 5.0$ amino acid protonation states ( $\mathrm{pH}$ of crystallization) using the empirical $\mathrm{pK}_{a}$ predictor, PROPKA, ${ }^{29}$ and removing the heme group from myoglobin. The apomyoglobin molecule was solvated in a cubic simulation box of dimensions $\mathrm{X}=\mathrm{Y}=\mathrm{Z}=7.1961 \mathrm{~nm}$ with $11613 \mathrm{SPC} / \mathrm{E}$ water molecules $^{30}$ applying periodic boundary conditions. Twelve $\mathrm{Cl}^{-}$counterions were added to neutralize the system. During all simulations, the LINCS ${ }^{31}$ algorithm was used to constrain all covalent bonds, while electrostatic interactions were calculated using the reaction field method. ${ }^{32}$ van der Waals interactions have been computed within a cutoff of $1.2 \mathrm{~nm}$. The MD integration time step was $2 \mathrm{fs}$. The protein volume (i.e., Voronoi volume) and protein cavities were calculated using the computational tools trjVoronoi $i^{33,34}$ and trj_cavity, ${ }^{35}$ respectively, both implemented in Gromacs.

Initially, the system was optimized following an energy minimization process using the steepest descent method during 5000 steps. Subsequently, the systems were simulated in an NpT ensemble for $100 \mathrm{~ns}$ at $\mathrm{P}=0.1 \mathrm{MPa}$ and $\mathrm{T}=300 \mathrm{~K}$ using the velocity rescale thermostat ${ }^{36}$ (coupling constant $\tau$ $=0.1 \mathrm{ps}$ ) and the Berendsen barostat ${ }^{37}$ (coupling constant $\tau=1.0 \mathrm{ps}$ ) for temperature and pressure control, respectively. After stabilization of the system $(100 \mathrm{~ns})$, the final configuration of this stage was taken as the starting point for the simulations under pressure. Thus, the system was coupled to a hydrostatic pressure bath, and the pressure was increased at regular increments of $10 \mathrm{MPa}$ each until a final pressure of $400 \mathrm{MPa}$ was achieved. Increments occurred systematically every $10 \mathrm{~ns}$ (an equilibration run of $5 \mathrm{~ns}$ and a collection period of $5 \mathrm{~ns}$ were performed for each pressure stage).

\section{A. Essential dynamics}

\section{Principal component analysis}

Collective coordinates, obtained as result of a principal component analysis (PCA) of atomic fluctuations, are commonly used to predict a low-dimensional subspace in which the essential protein motion is expected to take place. ${ }^{38} \mathrm{~A}$ covariance matrix based on fluctuations of main-chain atoms is diagonalized to generate eigenvectors and eigenvalues, which describe collective modes of fluctuation of the protein. ${ }^{39}$ Sorting the eigenvectors by the magnitude of the corresponding eigenvalues shows that the configurational space can be divided into a lower dimensional essential subspace in which most of the positional fluctuations are confined. ${ }^{40}$ Thus, according to the PCA method, each element of the covariance matrix $C$ can be represented as ${ }^{39,41}$

$$
\mathrm{C}_{i, j}=\left\langle x_{i}-\left\langle x_{i}\right\rangle\right\rangle\left\langle x_{j}-\left\langle x_{j}\right\rangle\right\rangle,
$$

where $x_{1}, \ldots, x_{3 \mathrm{~N}}$ are the mass-weighted Cartesian coordinates of an $\mathrm{N}$-particle system and \langle\rangle represents the average over all instantaneous structures sampled during the simulations. The symmetric $3 \mathrm{~N} \times 3 \mathrm{~N}$ matrix $\mathrm{C}$ can be diagonalized with an orthonormal transformation matrix $\mathrm{T}, q=\mathrm{T}^{\mathrm{T}}(x$ $-\langle x\rangle)$, which transforms $C$ into a diagonal matrix $\Lambda=\left\langle q q^{\mathrm{T}}\right\rangle$ of eigenvalues $\lambda_{i}$

$$
\Lambda=\mathrm{T}^{\mathrm{T}} \mathrm{CT}=\operatorname{diag}\left(\lambda_{1}, \lambda_{2}, \ldots, \lambda_{3 \mathrm{~N}}\right),
$$

where $\lambda_{1} \geq \lambda_{2} \geq \ldots \geq \lambda_{3 N}$. The $i$ th column of $\mathrm{T}$ is the eigenvector belonging to $\lambda_{i}$. Thus, the MD trajectory can be projected on the eigenvectors to determine the principal components (PC) $q_{i}(t), i=1, \ldots, 3 \mathrm{~N}$.

The first few PCs typically describe collective global motions of the system, with the first PC containing the largest mean-square fluctuation. Our covariance matrix was calculated using the $\mathrm{C} \alpha$ carbons from the apomyoglobin during the total time of the trajectory at $0.1 \mathrm{MPa}$ and under pressure scaling from 0.1 to $400 \mathrm{MPa}$.

\section{B. Free energy landscapes}

PCA is particularly useful in providing collective reaction coordinates for the construction of folding/unfolding free energy landscapes (FEL) of proteins. ${ }^{38}$ The FEL was computed as a function of the essential eigenvectors of motions to achieve two-dimensional representations $\left(q_{i}\right.$ and $q_{j}$ reaction coordinate) defined as 


$$
\mathrm{G}_{\alpha}=-\mathrm{K}_{\mathrm{B}} \mathrm{T} \ln \left[\frac{\mathrm{P}\left(q_{\alpha}\right)}{\mathrm{P}_{\mathrm{Max}}(q)}\right],
$$

where $K_{B}$ is the Boltzmann constant, $T$ is the temperature of simulation, and $G_{\alpha}$ is the free energy associated with the probability of finding the system in a particular state $q_{\alpha}$. Therefore, $\mathrm{P}\left(q_{\alpha}\right)$ is an estimate of the probability density function obtained from a histogram of the MD data, and $P_{\text {Max }}(q)$ is the probability of the most probable state.

\section{RESULTS}

\section{A. Analysis of protein cavity and volume variation}

After the exclusion of the heme group from the myoglobin, the cavity encompassed by G25, I28, L29, L32, T39, K42, F43, L72, L89, H93, I99, Y103, L104, F106, I107, S108, A110, I111, L135, and F138 residues evolved in time during 100 ns of the trajectory at $0.1 \mathrm{MPa}$ (Fig. 1), resulting in an average volume of $97.25 \pm 47.87 \AA^{3}$. However, when the apomyoglobin was subjected to increasing hydrostatic pressure, the internal cavity reduced $95.98 \%$ of its original value at pressures close to $50 \mathrm{MPa}$ (Fig. 2).

It is noteworthy that the addition of a small pressure increment resulted in a significant reduction of the cavity volume in the protein and that the slight variations observed under higher pressures can be assigned to the water penetration into the cavity upon exceeding $100 \mathrm{MPa}$ [Fig. 2(b)]. Our results suggest that at values below $100 \mathrm{MPa}$, the pressure would act mainly to compress the protein. At the same time, the probability of water penetration into the cavity appears to decrease with increase in the pressure. Moreover, volume changes in the cavity only indicated a loss of the local conformational order in the protein. Variations in the volume $\Delta \mathrm{V}$ $=V_{f}-V_{i}$, where those of the apomyoglobin under pressure were in agreement with the available experimental data ${ }^{1,43}$

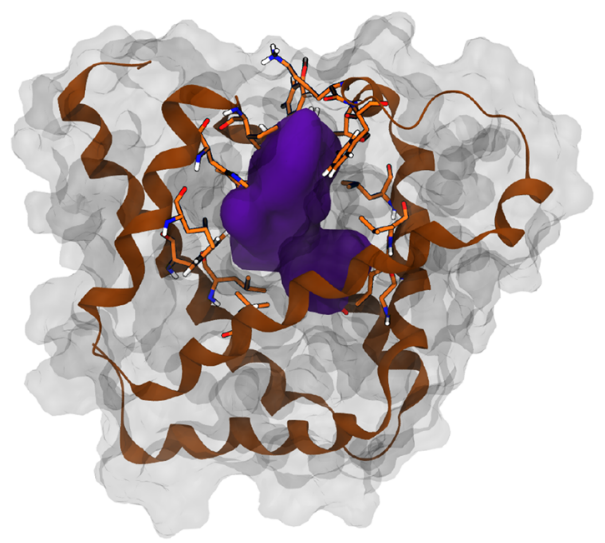

FIG. 1. Cavity in the apomyoglobin (in violet) surrounded by $\mathrm{G} 25, \mathrm{I} 28, \mathrm{~L} 29, \mathrm{~L} 32$, T39, K42, F43, L72, L89, H93, 199, Y103, L104, F106, I107, S108, A110, I111, $\mathrm{L} 135$, and $\mathrm{F} 138$ residues $(\mathrm{C}, \mathrm{H}, \mathrm{O}$, and $\mathrm{N}$ in orange, white, red, and blue, respectively). The protein surface in gray and the orange cartoon representation show the secondary structure. The figure was made using Visual Molecular Dynamics (VMD) program. ${ }^{42}$
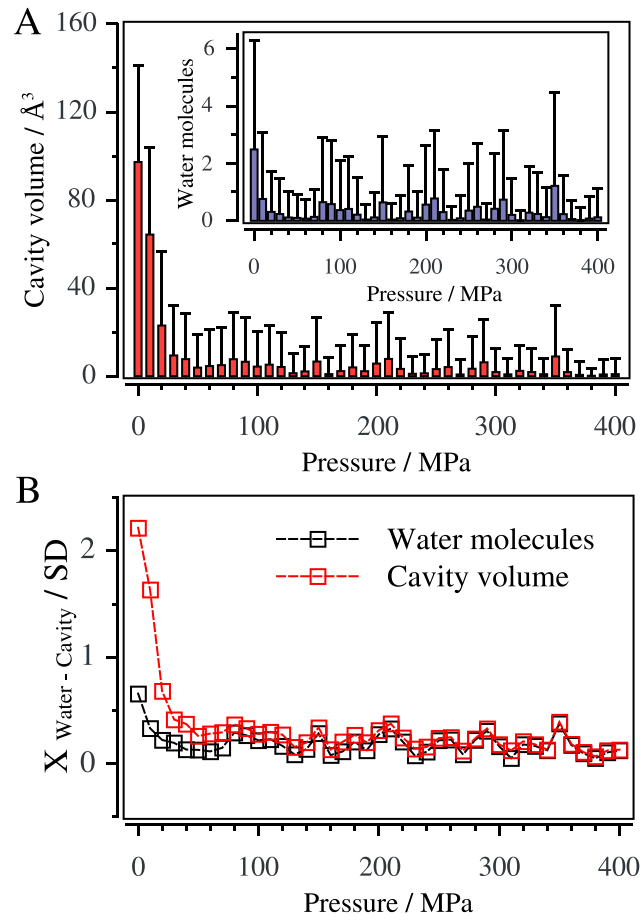

FIG. 2. Effects of the hydrostatic pressure on the cavity. (a) cavity volume and (inset) water molecules in the cavity for each pressure increase. Columns and error bars indicate the average and standard deviation, respectively. (b) the data were normalized with the standard deviation (SD) values in (a). Water penetration was computed as the number of solvent molecules fulfilling the cavity found for each frame. The variable $X$ represents either water molecules or the cavity volume, as applicable.

and our results showed that the $\Delta \mathrm{V}$ decreases with applied pressure, becoming negative (Fig. 3). According to Le Chatelier's principle, it is well known that increasing pressure favors configurations of smaller volume, ${ }^{44}$ indicating that the volume change upon unfolding is negative $\left(\Delta V_{u}\right)$, i.e., the specific molar volume of the unfolded state is smaller than that of the folded one. ${ }^{3}$

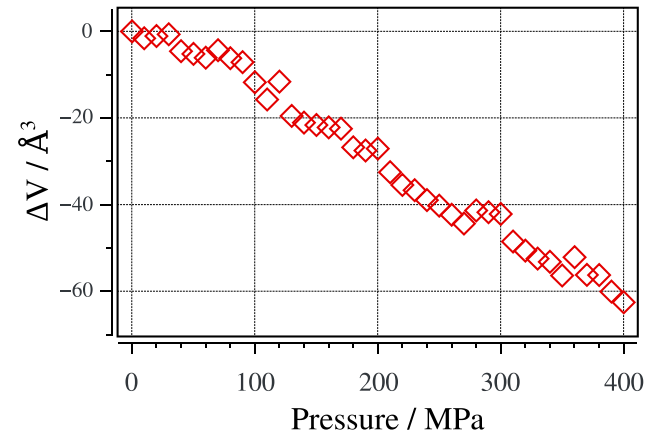

FIG. 3. Variation of protein volume under the pressure regime. $\Delta V=V_{f}-V_{i}$, where $V_{i}$ is the reference volume of the apomyoglobin in its native state at $0.1 \mathrm{MPa}$, and $V_{f}$ is the volume at each pressure coupling. 


\section{B. Solvent accessible surface area}

To check if water molecules invade the inner part of the protein, we analyzed the solvent accessible surface area (SASA) of apomyoglobin at each pressure coupling. Interestingly, the total SASA of the protein decreases with increasing pressure [Fig. 4(a)]. However, unlike volume variation (Fig. 3), negative values of SASA $\left(\Delta A_{\text {total }}=A_{f}-A_{i}\right)$ indicates that during pressure coupling, the surface area does not decrease linearly with increasing pressure. After achieving $100 \mathrm{MPa}$, an oscillatory behavior can be observed but never surpassing the reference value set as $0.1 \mathrm{MPa}$ [Fig. 4(a)].

In fact, total, hydrophilic and hydrophobic SASA values display a different trend above and below $200 \mathrm{MPa}$ [Figs. 4(a)-4(c)], respectively. For example, hydrophilic SASA [Fig. 4(b)] tends to increase when the pressure exceeds $200 \mathrm{MPa}$, while hydrophobic SASA acts contrariwise [Fig. 4(c)]. Our result suggests that the apomyoglobin produced locally disordered conformers due to variations in SASA due to the pressure increase. To check this issue, we computed the ratio between the hydrophobic/hydrophilic SASA and the total SASA for each pressure coupling. SASA fluctuations, particularly in the range of $0.1-400 \mathrm{MPa}$, rise as a consequence of different variations in the solvation state of hydrophobic and hydrophilic residues of the apomyoglobin [Fig. 4(d)].

Furthermore, it is noteworthy that above $200 \mathrm{MPa}$, hydrophilic and hydrophobic SASA contents present opposite trends, i.e., while one increases the other acts contrarily. However, total SASA seems to be more correlated with variations of the hydrophobic SASA [Figs. 4(b) and 4(c)], which represents $58 \%$ of the total area, instead of the hydrophilic one, presenting just $42 \%$. Further description is given in Sec. III F, where water penetration can be observed in Fig. 10.

\section{Principal component analysis}

To analyze how the system responded to the action of the increasing pressure, we also monitored the dynamical evolution to identify correlated movements by studying the MD trajectories of the $\mathrm{C} \alpha$ atoms from apomyoglobin using principal component analysis (PCA). For obtaining the principal components (PCs) and defining the essential subspaces (Sec. II: Computational methods) in the apomyoglobin, we calculated the projections of each trajectory onto their first five eigenvectors with largest amplitudes, i.e., eigenvalues (Fig. 1S in the supplementary material). For apomyoglobin at $0.1 \mathrm{MPa}$ and under pressure scaling, the top five components with the largest amplitudes represented $75.39 \%$ and $68.01 \%$ of the movements, respectively.

Thus, we could correlate the amplitude of the protein movements with the fluctuations of their residues and analyze local disorder of the system. We also computed the root mean square fluctuation (RMSF) of the $\mathrm{C} \alpha$ atoms of each apomyoglobin residue, projecting each trajectory $(0.1 \mathrm{MPa}$ and pressure coupling) onto their first five eigenvectors (Figs. 5 and $2 \mathrm{~S}$ in the supplementary material), to determine the relationship between local disorders of the system and their hydration profiles.

In both conditions, at $0.1 \mathrm{MPa}$ and under pressure scaling, $\mathrm{PC} 1$ exhibited the most significant fluctuations. When the protein was under pressure scaling, no substantial fluctuation was found in any of the five PCs examined when compared with those corresponding to $0.1 \mathrm{MPa}$ (Fig. $2 \mathrm{~S}$ in the supplementary material). In this particular case (pressure scaling), atomic mobility was restricted due to the increased pressure, the residues that showed the highest fluctuations being Val21 and His119 (Fig. 5) of the five PCs examined. We have selected these particular residues because they are located
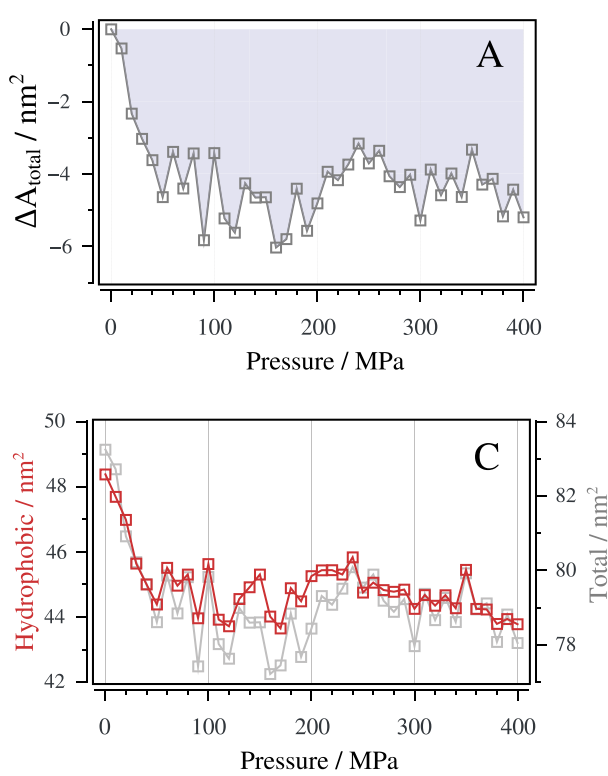
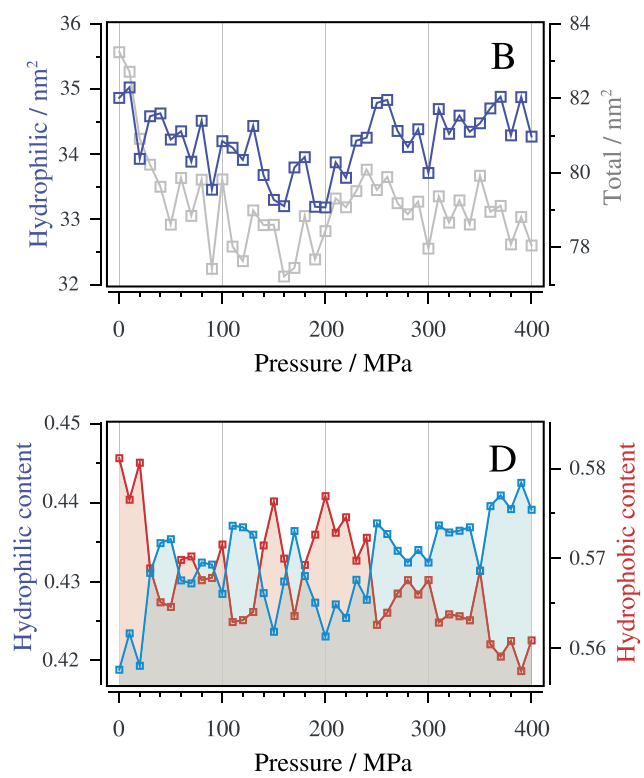

FIG. 4. Solvent accessible surface area (SASA) in apomyoglobin under hydrostatic pressures. In (a), $\Delta A_{\text {total }}=A_{f}-A_{i}$, where $A_{i}$ is the total reference area $(0.1$ $\mathrm{MPa}$ ) of the apomyoglobin in the native state, and $A_{f}$ is the total area at each pressure coupling. In (b), hydrophilic SASA (blue line) and total SASA (gray line). In (c), hydrophobic SASA (red line) and total SASA (gray line). In (d), the hydrophobic (red) and hydrophilic (blue) SASA content is represented as a ratio between the hydrophobic or hydrophilic SASA with the total SASA for each pressure coupling. In (b) and (c) total SASA is used as a graphical reference to analyze the behavior of the partial components, $A_{\text {total }}=A_{\text {hydrophilic }}+A_{\text {hydrophobic }}$. 
A

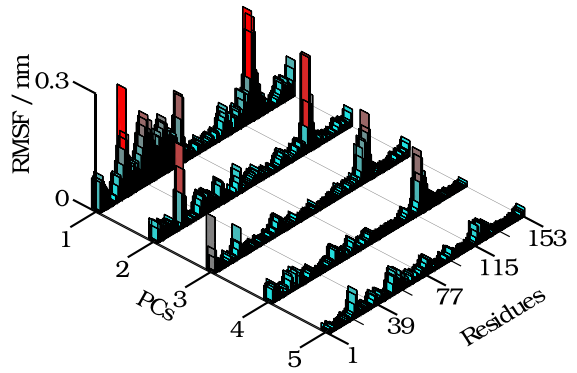

B

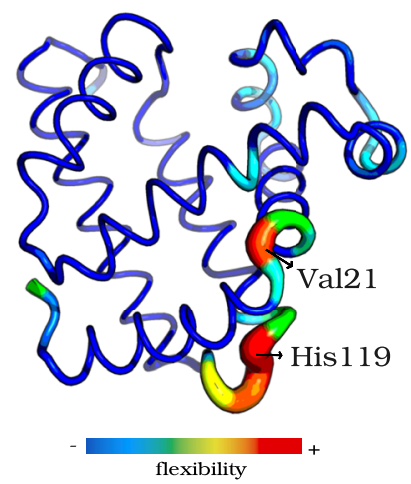

FIG. 5. (a) Root mean square fluctuation (RMSF) of the apomyoglobin under pressure scaling, projecting each trajectory onto their first five eigenvectors. In red, the residues with the greatest fluctuation Val21 and His119. (b) B-factor putty representation of PC1 shown in (a). The differential colors are according to the residue flexibility values. The thickness of the tube is proportional to the fluctuations.

on the surface of the protein and are part of an $\alpha$-turn that is more susceptible to be affected by the solvent effect. So, they can act as checkpoints to understand the solvent effect exerted on the protein under pressure.

\section{Hydrogen bond network}

Hydrogen bond (HB) network is structurally affected by pressure increase due to the rearrangement of water molecules surrounding the protein. So, we analyzed the pressure effect upon the global distribution of hydrogen bonds and the number of Donor-Acceptor (DA) pairs (Fig. 6) involving the formation of a hydrogen bond. $\mathrm{HB}$ and DA analyses are determined based on cutoffs for the hydrogendonor-acceptor angle $\left(30^{\circ}\right)$ and the donor-acceptor distance $(0.35 \mathrm{~nm})$ between the two groups involved (water-water or water-protein).

The relationship HB/DA is a measure of the hydrogen bond density as a function of the number of available DA pairs. It helps visualize how the network changes when normal conditions are affected. When apomyoglobin is subjected to increasing pressures, the HB/DA ratio decreases, indicating that water molecules reorganize themselves around the protein due to volume compression [Fig. 6(a)]. However, when the pressure exceeds $200 \mathrm{MPa}$, a discontinuity in the ratio HB/DA can be observed [Figs. 6(b) and 6(c)].
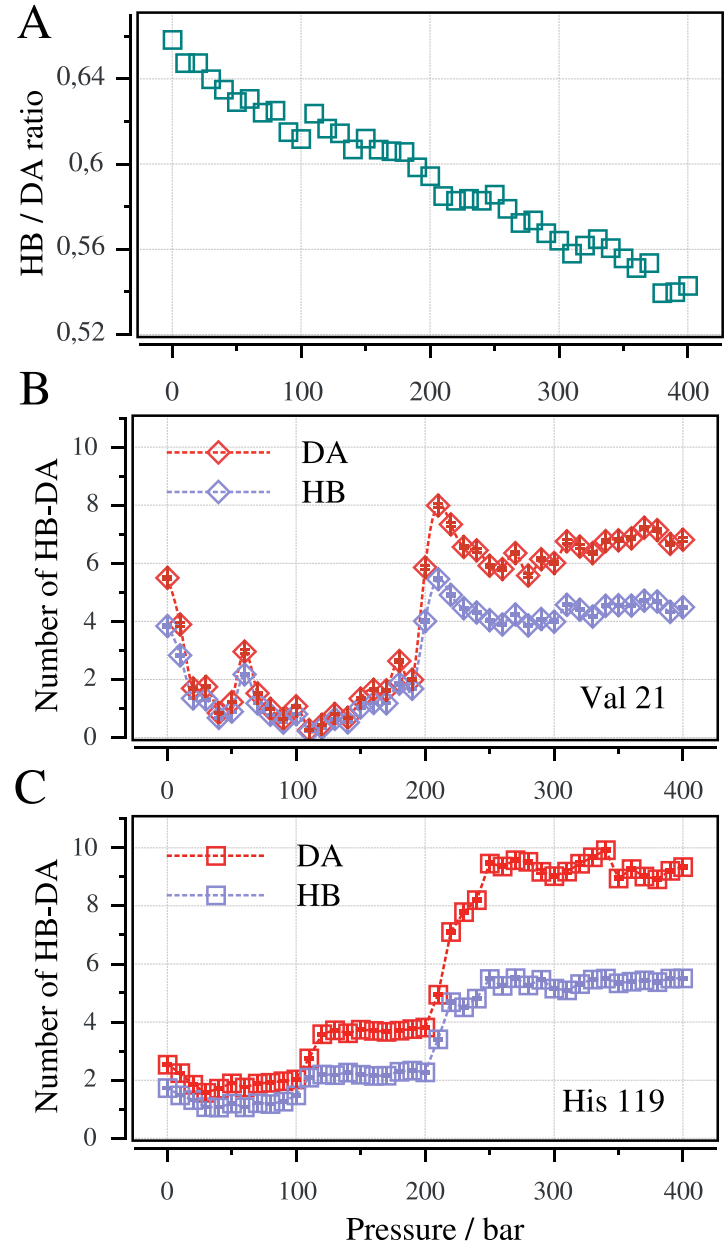

FIG. 6. Hydrogen bonds analysis. (a) the ratio between hydrogen bonds (HB) and Donor-Acceptor (DA) pairs of HB. The HB/DA ratio was computed between apomyoglobin-water. (b) number of HB (blue diamonds) and DA (red diamonds) around of Val21. (c) number of HB (blue squares) and DA (red squares) around of His119. For (b) and (c), only hydrogen bonds between water molecules within the shell distance of $0.5 \mathrm{~nm}$ from the $\mathrm{C} \alpha$ atom on Val21 and His 119 are considered. Squares and diamonds represent the average, and the error bars represent the standard deviations. In all, HB and DA analyses are determined based on cutoffs for the hydrogen-donor-acceptor angle $\left(30^{\circ}\right)$ and the donor-acceptor distance $(0.35 \mathrm{~nm})$.

We also observed that both Val21 and His119 show a different trend regarding the number of hydrogen bonds formed between water molecules within the shell of $0.5 \mathrm{~nm}$ radius from the $\mathrm{C} \alpha$ atom and these hydrophobic and hydrophilic residues, respectively.

It is noteworthy that around the hydrophobic residue Val21, in the 0.1-200 MPa range, the hydrophobic effect is favored: (i) the number of the DA pairs is proportional to HBs formed, and (ii) for the selected cutoff, the distance between water molecules (HB network) and Val21 increases, decreasing the HB and DA counts. The scenario is different for pressures $>200 \mathrm{MPa}$, where compression increased the number of water molecules around Val21 (more DA pairs available). Although 
the number of HBs increases, the HB network formation is less favored since for each available DA pair, it is not possible to form as many HBs as those formed under low pressures [Fig. 6(b)].

On the other hand, for the hydrophilic residue His119, the results are not conclusive about the relationship between DA pairs and HBs network under pressures from 0.1 to $200 \mathrm{MPa}$. Even between 100 and $200 \mathrm{MPa}$, an increase in DA pairs is evident without an apparent augmentation in the HBs number of water molecules, given that solvent molecules form hydrogen bonds with hydrophilic residues. However, similar to Val21, the number of water molecules around His119 increased at pressures $>200 \mathrm{MPa}$ due to the compression effect, but with a more significant number of HBs and DA pairs, mainly due to the hydrophilic environment [Fig. 6(c)].

\section{E. Water structure}

A key point to this phenomenon is the change in the behavior of water molecules when the pressure exceeds $200 \mathrm{MPa}$. In our simulations, we observed an increment of the number of water-water hydrogen bonds with increasing pressure, resulting in the restructuring of the hydrogen bond network around the protein and a change in the protein environment. In this regard, we analyzed the solvation dynamics calculating the density of water molecules in the

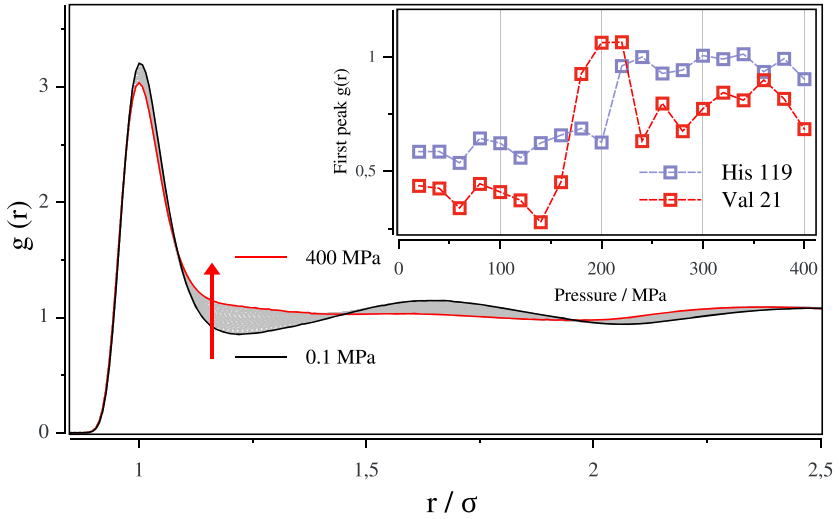

FIG. 7. Radial distribution function, $g(r)$, under pressure scaling between oxygen atoms of water molecules. Arrow indicates the direction the increased pressure. The normalization value refers to the diameter of a water molecule $(0.275 \mathrm{~nm})$. Inset: the first peak of $g(r)$ between oxygen atoms of water molecules around the hydrophilic (His 119) and hydrophobic (Val21) regions (C $\alpha$ atoms in the amino acid residues) in apomyoglobin at high pressures.

vicinity the hydrophobic and hydrophilic regions using the radial distribution function $g(r)$ between water oxygen atoms (Fig. 7).
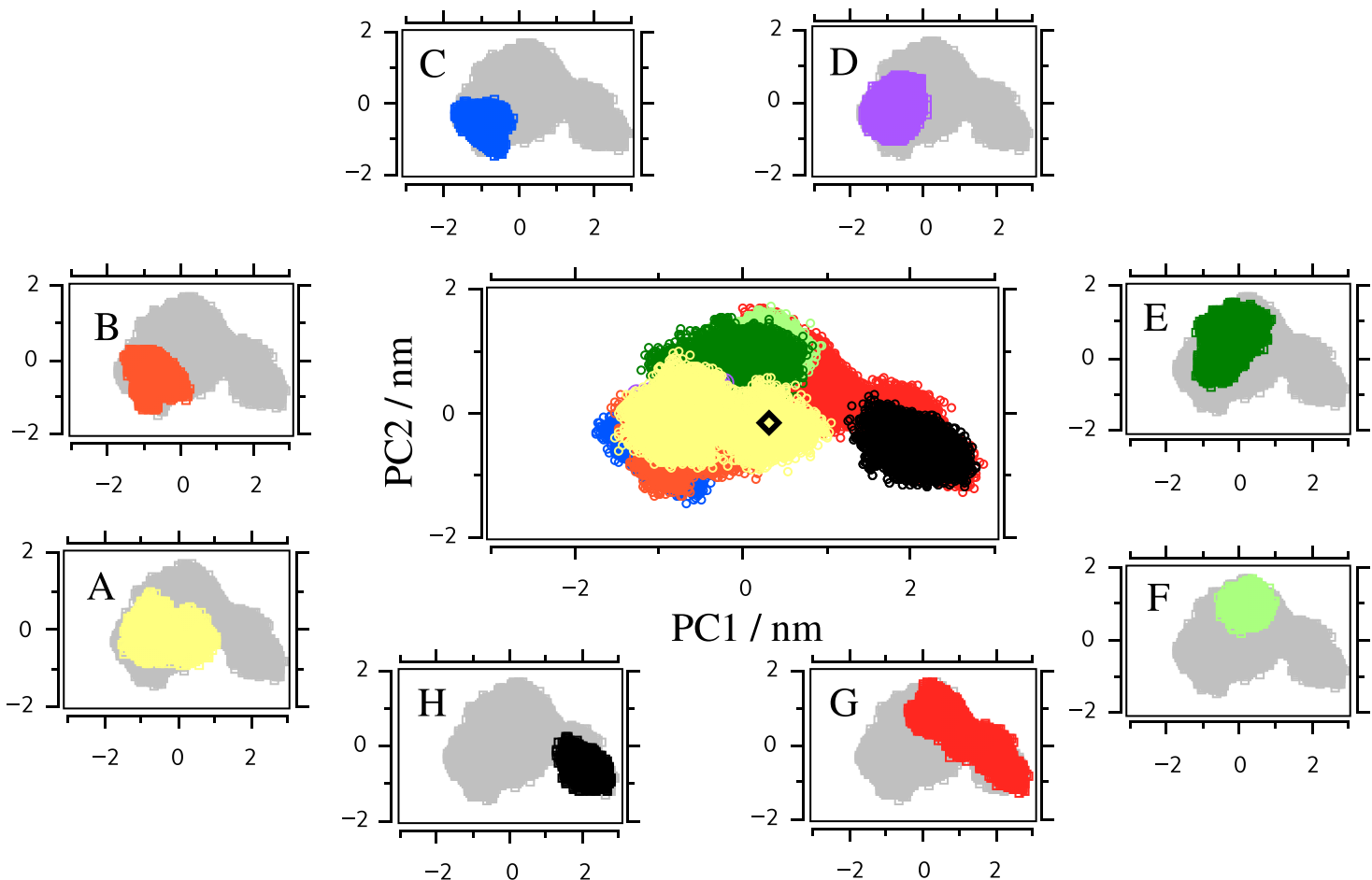

FIG. 8. Conformational sampling obtained by bi-dimensional (2D) projection of the trajectories onto the first two PCs. In the main box, the diamond shows the most probable structural conformations to $0.1 \mathrm{MPa}$. The smaller boxes indicate the protein substates for each pressure range analyzed: (a) $0.1-50 \mathrm{MPa}$, (b) $50-100 \mathrm{MPa}$, (c) $100-150 \mathrm{MPa}$, (d) 150-200 MPa, (e) 200-250 MPa, (f) 250-300 MPa, (g) 300-350 Mpa, (h) 350-400 Mpa. 
In general terms, each $g(r)$ peak shows the preferred position of the successive neighboring water coordination layers. ${ }^{16}$ According to the two-state model, in the classical shape of the $g(r)$ of water at $0.1 \mathrm{MPa}$, the second peak reveals the tetrahedral component of the water structure. Consequently, the increase in pressure induces an increase in the hexagonal structural contribution in detriment of the tetrahedral one. ${ }^{16,17}$

As pressure increases, while the position of the first peak remains unaltered, the position of the second peak is attenuated, being replaced by a valley, revealing the presence of a simple structure in the solvent (Fig. 7). However, an increase in pressure generated a change in the density of water molecules around the hydrophobic and hydrophilic regions (Fig. 7 inset).

\section{F. Free energy landscapes}

We analyzed the conformational changes in the apomyoglobin associated with the probability of finding the protein in a particular state under the effect of high pressures. Thus, with the calculation of the first two PCs, it is possible to recover more than half of the total fluctuation of apomyoglobin (Fig. 1S in the supplementary material). In Fig. 8, it is shown the probability distribution functions $p(q)$ of the first two PCs, where each point represents a transitional protein conformer. Therefore, when apomyoglobin is subjected to pressure enhancement, it migrates out of its most probable structural conformations at $0.1 \mathrm{MPa}$, experiencing a slight displacement from the native state towards a population of conformers at pressures in the 10-50 MPa range (Fig. 3S in the supplementary material) characterized by the loss of volume in the cavity (Fig. 2). These new conformers remained unaltered until an approximate value close to $200 \mathrm{MPa}$ [Figs. 8(a)-8(d)] was achieved. Above this threshold, the protein jumped off towards a new region of the conformational space between 200 and $300 \mathrm{MPa}$ [Figs. 8(e) and $8(\mathrm{f})$ ] and explored once again new regions at $300-400 \mathrm{MPa}$ [Figs. 8(g) and 8(h)].

Moreover, from $p(q)$ analysis, we can explore the free energy landscape by obtaining the probability density corresponding to the overall free energy minimum position of the apomyoglobin analyzing the new conformations achieved by the system at different pressures (see Sec. II).

The FEL analysis suggests that the system under the highpressure regime presents four conformational states defined

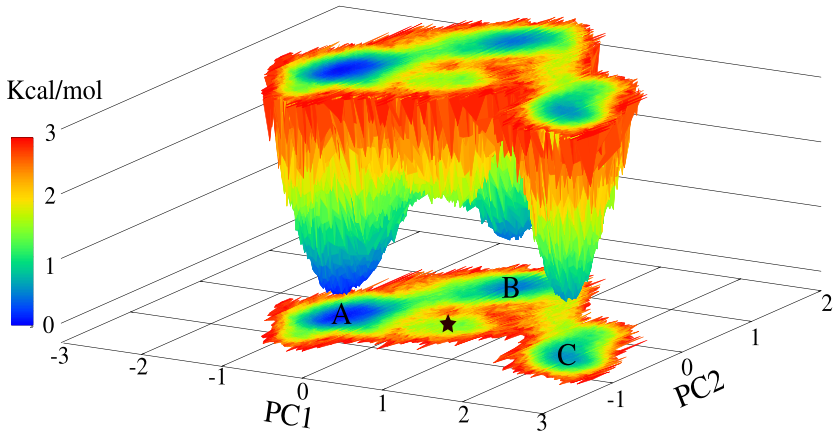

FIG. 9. Free energy landscape using as reaction coordinates the projection of the apomyoglobin under pressure increasing trajectory along the first two principal comportments. The star and $A, B$, and $C$ labels indicate the localization on the FEL of the most probable protein conformers (local minima) for pressure values of $0.1 \mathrm{MPa}, 10-200 \mathrm{MPa}, 200-350 \mathrm{MPa}$, and above $350 \mathrm{MPa}$, respectively (See Fig. 8).

by four minima (Fig. 9), indicating a progressive conformational transition towards different states. The first local minima correspond to conformers in the native state at $0.1 \mathrm{MPa}$. For pressures below $200 \mathrm{MPa}$, the protein abandons its native conformation and migrates toward a new state characterized (Fig. 8) by conformers lacking a defined cavity (second local minima at $0.07 \mathrm{kcal} \mathrm{mol}^{-1}$ ).

We observed that increasing pressures allows apomyoglobin to visit several regions of the FEL. We detected that the system visited a new conformation [Fig. 9(b)], different from the equilibrium stage at $0.1 \mathrm{MPa}$ when the threshold of $200 \mathrm{MPa}$ was overcome $\left(0.61 \mathrm{kcal} \mathrm{mol}^{-1}\right)$. Interestingly, at pressures between 300 and $350 \mathrm{MPa}$, the system jumped off this third minimum [Fig. 8(g)] and explored additional regions of the conformational space achieving the fourth minimum finally $\left(0.43 \mathrm{kcal} \mathrm{mol}^{-1}\right)$ at pressures above $350 \mathrm{MPa}$ [Fig. 8(h)]. Visual inspection of the lowest energy structure revealed that at $220 \mathrm{MPa}$, water molecules penetrate into the protein closer to the hydrophobic core. This water infiltration became even more evident at $400 \mathrm{MPa}$ (Fig. 10).

Thus, our outcomes indicate that the pressure induced local disorder, which can be strongly correlated with a transition from the native to the molten globule state. According to available experimental data, the production of a molten globule induced by pressure in apomyoglobin at $\mathrm{pH}$ 6-7 was reported to occur around $200 \mathrm{MPa} .^{22}$

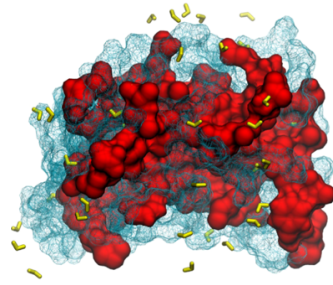

$0.1 \mathrm{MPa}$

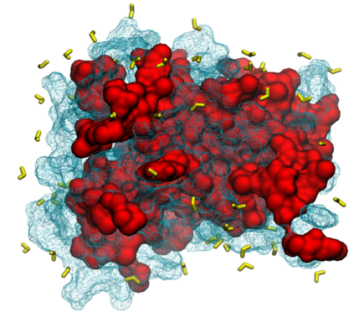

$220 \mathrm{MPa}$

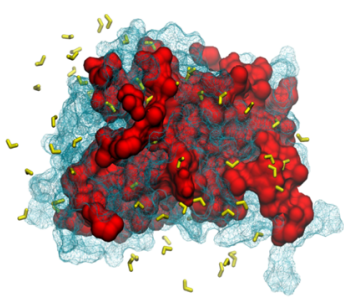

$400 \mathrm{MPa}$
FIG. 10. Lowest energy apomyoglobin conformers computed in Fig. 9. Hydrophobic and hydrophilic surface are in red and light blue, respectively. The water molecules (yellow) are represented for a cutoff of $1.4 \AA$ of the hydrophobic surface. 


\section{DISCUSSION}

An exciting debate arises when microscopic mechanisms involved in the denaturation of proteins at high pressures are explained. In particular, the issue emerges when the hydrophobic interaction is invoked, given that hydrophobicity cannot elucidate by itself the volume changes measured during protein unfolding. ${ }^{44}$ Thus, according to the hydrophobic model, if the transfer of hydrophobic compounds to water is accompanied by a significant negative volume change at low pressure,$^{45}$ then at high pressures, this volume change increases becoming positive. ${ }^{46,47}$ Consistently, Royer ${ }^{43}$ reported that in the case of the unfolding of proteins, $\Delta \mathrm{V}$ is negative at high pressures in most cases (Fig. 3). In other work, they performed a study in which they spawned 10 new variants of an internal cavity of staphylococcal nuclease. ${ }^{8}$ These variants had different volumes and with adding pressure to the system, the authors observed that during the protein unfolding the cavities were completely lost suggesting that destabilization was due to the cavities disruption, even at pressure values lower than $50 \mathrm{MPa}$.

Several theoretical and experimental studies demonstrate that pressure effects are evidenced locally and can be subtler than those produced by changes in temperature. ${ }^{8}$ Thus, alterations in the local environments of amino acid residues due to changes in the pressure lead to conformational fluctuations (locally disordered conformers) associated with an increase in the solvation state of the proteins. ${ }^{22,44,48,49}$

Another critical aspect of the hydrophobic model is the one which states that unfolding of proteins at high pressures produces the exposure of hydrophobic residues, formerly located at the inner part of proteins, to bulk water. ${ }^{43,44}$ Instead, Gregorio Weber has raised the hypothesis that under high pressures, proteins could be penetrated by water. ${ }^{7}$ Anyhow, in both cases, either with the exposition of hydrophobic residues to water or the solvation of inner residues by penetrating water, hydration of hydrophobic residues is favored by increasing pressure 22,50 (Figs. 4 and 10).

However, although both $\triangle \mathrm{SASA}$ and $\Delta \mathrm{V}$ decreases with increasing pressure, the volume decreases linearly (Fig. 3) while the SASA change its trend at specific pressures (Fig. 4). Therefore, modifications in the SASA provide relevant information in relation to the local conformational changes in the protein.

Moreover, the inner cavity becomes more susceptible to compression (Fig. 2). In this regard, some NMR and fluorescence spectroscopy studies have shown that different regions of the apomyoglobin can act differently when compressed, being more or less susceptible to changes with pressure. ${ }^{22,48}$

Hence, in agreement with experimental data, ${ }^{18}$ our results suggest that changes in the hydration shell around the protein could be mainly due to the pressure effect on the hydrogen bonding network of water (Fig. 6). The first hydration shell of $\mathrm{H}$-bonds would be almost unaffected by pressure (Fig. 7), while molecules in the vicinity of the second-shell would approach the interstitial sites of the first tetrahedral shell as the pressure increases giving rise to a hexagonal structure. ${ }^{16}$

In other words, the structure of water can be represented as a combination of two different local structures, called lowdensity water (LDW, open tetrahedral structure) and highdensity water (HDW, more compact hexagonal structure). ${ }^{16-18}$ Thus, under the pressure regime above $200 \mathrm{MPa}$, the liquid water shifts from a low-density state to a high-density state, favoring the upsurge in the solvation of the hydrophilic and hydrophobic residues. It is imperative to emphasize that so far, the only water model representing the liquid-liquid transition (LWD to HWD) at the pressure experimentally reported $(\sim 0.2$ $\mathrm{GPa})^{16,18}$ is the SPC/E model.

Both, the hydrophobic and hydrophilic solvation depend on thermodynamic conditions of the solvent (e.g., pressuretemperature). ${ }^{51}$ Thus, changes in the free energy of the system are due to structural and energetic changes in the solvent around each solute molecule, ${ }^{51,52}$ and hence, water molecules exhibits a preferred orientation at the hydrophilic or hydrophobic surface. ${ }^{53}$ This is linked to the result of Djikaev and Ruckenstein, ${ }^{51}$ who computed the effective width of the fluid solvent-solute transition layer $(\sigma)$ and average density $(\rho)$ therein, where the behavior change of $\sigma$ and $\rho$ modifies the hydration mechanism depending on the solute-solvent affinity. Similarly, Luzar and co-workers ${ }^{53}$ concluded that in the average number of hydrogen bonds per molecule, only the nearest neighbor interactions among water molecules confined to the length of the $\mathrm{O}-\mathrm{H} \cdots \mathrm{O}$ bond are taken into account, due to more distant water molecules are less important in the behavior of the average number of hydrogen bonds in the solvent-solute interface.

Our analysis suggests that a liquid-liquid transition in water favors the solvation of apomyoglobin, which leads us to believe that the conformational changes suffered by the protein could be correlated with the structural changes in the hydration pattern. Also, some small Angle X-ray scattering experiments show that the reduction of motions at high pressure can be related to an increase of the density in the hydration shell of proteins. ${ }^{54}$

Hence, our data indicate that the conformational changes in the protein are accompanied by a qualitative change in the hydration water shell dynamics involving locally disordered conformers produced by water diffusion from the bulk to the inner portion of the protein. Thus, the mechanism of pressure denaturation can be related to the disruption of the hydrogenbond network of water favoring the coexistence of two states, low-density and high-density water, which directly implies in the formation of a molten globule once the threshold of 200 MPa has been overcome.

\section{CONCLUSIONS}

In this work, we investigate the effect of the pressure in the apomyoglobin generated from the removal of the heme group of the whale sperm myoglobin using MD and PCA as analysis tools.

Our data showed a correlation between SASA and the volume determined by the decreasing tendency in both with 
increase the pressure. However, unlike the volume, the SASA shows changes in the solvation dynamics of the polypeptide chain, observing a heterogeneous hydration due to fluctuations in water density.

Thus, for pressures lower than the point of liquid-liquid transition in water (LWD to HWD), the most probable conformational states in the protein are characterized by a drastic reduction in the cavity volume, leading to a decrease in the number of water molecules inside these, due to the lack of available volume to occupy. However, once the pressure exceeds the liquid-liquid transition point, a small fraction of water molecules hydrate this region. Therefore, lowest energy apomyoglobin conformer observed in our simulations, is the one that refers to conformations lacking cavity and sensitive to compression.

For pressure values above the LWD to HWD transition point, the structural changes in the hydrogen bond network result in the process of gradual weakening of the hydrophobic effect. The water structure is highly influenced by pressure, generating structural anomalies in the liquid water, the second coordination shell collapses on to the first coordination shell and the water density is modified (at this stage, water acts as an unstructured liquid), leading to an increase in the hydration of the apomyoglobin caused by the insertion of water molecules inside the protein. Finally, we can conclude that the main driving force of protein denaturation at high pressures is an increase in its hydration state inducing in the apomyoglobin a conformational change where the internal cavity was largely reduced to increase the population of less ordered conformers, resulting in the formation of a molten globule.

\section{SUPPLEMENTARY MATERIAL}

See supplementary material for additional information on the cumulative percentage contribution of the total motions in each system, RMS fluctuations of the $\mathrm{C} \alpha$ atoms of each apomyoglobin residue, and the analyzed $\mathrm{PC} 1$ and $\mathrm{PC} 2$ bidimensional projection (2D) of the trajectories for each pressure.

\section{ACKNOWLEDGMENTS}

This work was funded by the National Research Council of Argentina (CONICET).

\section{REFERENCES}

${ }^{1}$ J. L. Silva, A. C. Oliveira, T. C. Vieira, G. A. de Oliveira, M. C. Suarez, and D. Foguel, "High-pressure chemical biology and biotechnology," Chem. Rev. 114, 7239-7267 (2014)

2J. L. Silva, D. Foguel, and C. A. Royer, "Pressure provides new insights into protein folding, dynamics and structure," Trends Biochem. Sci. 26, 612-618 (2001).

${ }^{3}$ J. Roche, C. A. Royer, and C. Roumestand, "Monitoring protein folding through high pressure NMR spectroscopy," in Progress in Nuclear Magnetic Resonance Spectroscopy," Prog. Nucl. Magn. Reson. Spectrosc. 102-103, 15-31 (2017)

${ }^{4} \mathrm{~K}$. Akasaka, "Exploring the entire conformational space of proteins by highpressure NMR," Pure Appl. Chem. 75, 927-936 (2003).
${ }^{\mathbf{5}} \mathrm{K}$. Akasaka, "Protein studies by high-pressure NMR," in Experimental Approaches of NMR Spectroscopy (Springer, 2018), pp. 3-36.

${ }^{6}$ L. M. Nguyen and J. Roche, "High-pressure NMR techniques for the study of protein dynamics, folding and aggregation," J. Magn. Reson. 277, 179-185 (2017).

${ }^{7}$ C. R. Chen and G. I. Makhatadze, "Molecular determinant of the effects of hydrostatic pressure on protein folding stability," Nat. Commun. 8, 14561 (2017).

${ }^{8}$ J. Roche, J. A. Caro, D. R. Norberto, P. Barthe, C. Roumestand, J. L. Schlessman, A. E. Garcia, C. A. Royer et al., "Cavities determine the pressure unfolding of proteins," Proc. Natl. Acad. Sci. U. S. A. 109, 6945-6950 (2012).

${ }^{9}$ J. A. Caro and A. J. Wand, "Practical aspects of high-pressure NMR spectroscopy and its applications in protein biophysics and structural biology," Methods 148, 67-80 (2018).

${ }^{10}$ B. Guignon, E. H. Baltasar, P. D. Sanz, V. G. Baonza, and M. Taravillo, "Evidence of low-density water to high-density water structural transformation in milk during high-pressure processing," Innovative Food Sci. Emerging Technol. 38, 238-242 (2016)

${ }^{11}$ Y. R. Espinosa, J. R. Grigera, and E. R. Caffarena, "Essential dynamics of the cold denaturation: Pressure and temperature effects in yeast frataxin," Proteins: Struct., Funct., Bioinf. 85, 125-136 (2017).

${ }^{12}$ J. R. Grigera and A. N. McCarthy, "The behavior of the hydrophobic effect under pressure and protein denaturation," Biophys. J. 98, 1626-1631 (2010).

${ }^{13}$ J.-B. Rouget, T. Aksel, J. Roche, J.-L. Saldana, A. E. Garcia, D. Barrick, and C. A. Royer, "Size and sequence and the volume change of protein folding," J. Am. Chem. Soc. 133, 6020-6027 (2011).

${ }^{14}$ G. Hummer, S. Garde, A. E. García, M. E. Paulaitis, and L. R. Pratt, "The pressure dependence of hydrophobic interactions is consistent with the observed pressure denaturation of proteins," Proc. Natl. Acad. Sci. U. S. A. 95, 1552-1555 (1998).

${ }^{15}$ J. R. Errington and P. G. Debenedetti, "Relationship between structural order and the anomalies of liquid water," Nature 409, 318 (2001).

${ }^{16}$ O. Chara, A. N. McCarthy, and J. R. Grigera, "Crossover between tetrahedral and hexagonal structures in liquid water," Phys. Lett. A 375, 572-576 (2011).

${ }^{17}$ A. K. Soper and M. A. Ricci, "Structures of high-density and low-density water," Phys. Rev. Lett. 84, 2881 (2000).

${ }^{18}$ S. Fanetti, A. Lapini, M. Pagliai, M. Citroni, M. Di Donato, S. Scandolo, R. Righini, and R. Bini, "Structure and dynamics of low-density and highdensity liquid water at high pressure," J. Phys. Chem. Lett. 5, 235-240 (2013).

${ }^{19}$ Y. R. Espinosa, E. R. Caffarena, Y. B. Martínez, and J. R. Grigera, "Pressure effect on micellization of non-ionic surfactant Triton X-100," J. Chem. Phys. 148, 074901 (2018).

${ }^{20} \mathrm{D}$. Zhang and R. Lazim, "Application of conventional molecular dynamics simulation in evaluating the stability of apomyoglobin in urea solution," Sci. Rep. 7, 44651 (2017).

${ }^{21}$ A. Onufriev, D. A. Case, and D. Bashford, "Structural details, pathways, and energetics of unfolding apomyoglobin," J. Mol. Biol. 325, 555-567 (2003).

${ }^{22}$ R. Kitahara, H. Yamada, K. Akasaka, and P. E. Wright, "High pressure NMR reveals that apomyoglobin is an equilibrium mixture from the native to the unfolded," J. Mol. Biol. 320, 311-319 (2002).

${ }^{23}$ F. Librizzi, R. Carrotta, J. Peters, and A. Cupane, "The effects of pressure on the energy landscape of proteins," Sci. Rep. 8, 2037 (2018).

${ }^{24}$ H. J. Dyson and P. E. Wright, "How does your protein fold? Elucidating the apomyoglobin folding pathway," Acc. Chem. Res. 50, 105-111 (2016).

${ }^{25}$ D. Van Der Spoel, E. Lindahl, B. Hess, G. Groenhof, A. E. Mark, and H. J. Berendsen, "GROMACS: Fast, flexible, and free," J. Comput. Chem. 26, 1701-1718 (2005).

${ }^{26}$ B. Hess, C. Kutzner, D. Van Der Spoel, and E. Lindahl, "GROMACS 4: Algorithms for highly efficient, load-balanced, and scalable molecular simulation," J. Chem. Theory Comput. 4, 435-447 (2008). 
${ }^{27}$ W. R. P. Scott, P. H. Hünenberger, I. G. Tironi, A. E. Mark, S. R. Billeter, J. Fennen, A. E. Torda, T. Huber, P. Krüger, and W. F. van Gunsteren, "The GROMOS biomolecular simulation program package," J. Phys. Chem. A 103, 3596-3607 (1999).

${ }^{28} \mathrm{~F}$. Yang and G. N. Phillips, Jr., "Crystal structures of $\mathrm{CO}^{-}$, deoxy- and metmyoglobins at various $\mathrm{pH}$ values," J. Mol. Biol. 256, 762-774 (1996).

${ }^{29}$ M. H. Olsson, C. R. Søndergaard, M. Rostkowski, and J. H. Jensen, "PROPKA3: Consistent treatment of internal and surface residues in empirical $\mathrm{pK}_{\mathrm{a}}$ predictions," J. Chem. Theory Comput. 7, 525-537 (2011).

${ }^{30}$ H. J. C. Berendsen, J. R. Grigera, and T. P. Straatsma, "The missing term in effective pair potentials," J. Phys. Chem. 91, 6269-6271 (1987).

${ }^{31}$ B. Hess, H. Bekker, H. J. C. Berendsen, and J. G. E. M. Fraaije, "LINCS: A linear constraint solver for molecular simulations," J. Comput. Chem. 18, 1463-1472 (1997).

${ }^{32}$ W. F. van Gunsteren, H. J. Berendsen, and J. A. Rullmann, "Inclusion of reaction fields in molecular dynamics. Application to liquid water," Faraday Discuss. Chem. Soc. 66, 58-70 (1978).

${ }^{33} \mathrm{C}$. Rycroft, "VORO++: A three-dimensional Voronoi cell library in $\mathrm{C}++, "$ Chaos 19, 041111 (2009).

${ }^{34}$ S. Abel, F.-Y. Dupradeau, and M. Marchi, "Molecular dynamics simulations of a characteristic DPC micelle in water," J. Chem. Theory Comput. 8, 46104623 (2012)

${ }^{35}$ T. Paramo, A. East, D. Garzoìn, M. B. Ulmschneider, and P. J. Bond, "Efficient characterization of protein cavities within molecular simulation trajectories: trj_cavity," J. Chem. Theory Comput. 10, 2151-2164 (2014).

${ }^{36}$ G. Bussi, D. Donadio, and M. Parrinello, "Canonical sampling through velocity rescaling," J. Chem. Phys. 126, 014101 (2007).

${ }^{37}$ H. J. C. Berendsen, J. P. M. Postma, W. F. van Gunsteren, A. DiNola, and J. R. Haak, "Molecular dynamics with coupling to an external bath," J. Chem. Phys. 81, 3684-3690 (1984).

${ }^{38}$ I. Daidone and A. Amadei, "Essential dynamics: Foundation and applications," Wiley Interdiscip. Rev.: Comput. Mol. Sci. 2, 762-770 (2012).

${ }^{39}$ A. Amadei, A. B. M. Linssen, and H. J. C. Berendsen, "Essential dynamics of proteins," Proteins: Struct., Funct., Genet. 17, 412-425 (1993).

${ }^{40}$ I. Daidone, A. Amadei, and A. Di Nola, "Thermodynamic and kinetic characterization of a $\beta$-hairpin peptide in solution: An extended phase space sampling by molecular dynamics simulations in explicit water," Proteins: Struct., Funct., Bioinf. 59, 510-518 (2005).
${ }^{41}$ G. G. Maisuradze, A. Liwo, and H. A. Scheraga, "Relation between free energy landscapes of proteins and dynamics," J. Chem. Theory Comput. 6 , 583-595 (2010).

${ }^{42}$ W. Humphrey, A. Dalke, and K. Schulten, "VMD: Visual molecular dynamics," J. Mol. Graphics 14, 33-38 (1996).

${ }^{43} \mathrm{C}$. A. Royer, "Revisiting volume changes in pressure-induced protein unfolding," Biochim. Biophys. Acta, Protein Struct. Mol. Enzymol. 1595, 201-209 (2002).

${ }^{44} \mathrm{C}$. L. Dias, "Unifying microscopic mechanism for pressure and cold denaturations of proteins," Phys. Rev. Lett. 109, 048104 (2012).

${ }^{45} \mathrm{~W}$. d. Masterton, "Partial molal volumes of hydrocarbons in water solution," J. Chem. Phys. 22, 1830-1833 (1954).

${ }^{46} \mathrm{~W}$. Kauzmann, "Thermodynamics of unfolding," Nature 325, 763-764 (1987).

${ }^{47}$ S. Sawamura, K. Kitamura, and Y. Taniguchi, "Effect of pressure on the solubilities of benzene and alkylbenzenes in water," J. Phys. Chem. 93, 49314935 (1989).

${ }^{48}$ N. Tanaka, C. Ikeda, K. Kanaori, K. Hiraga, T. Konno, and S. Kunugi, "Pressure effect on the conformational fluctuation of apomyoglobin in the native state," Biochemistry 39, 12063-12068 (2000).

${ }^{49}$ M. D. Collins, G. Hummer, M. L. Quillin, B. W. Matthews, and S. M. Gruner, "Cooperative water filling of a nonpolar protein cavity observed by highpressure crystallography and simulation," Proc. Natl. Acad. Sci. U. S. A. 102, 16668-16671 (2005).

${ }^{50}$ S. Sarupria, T. Ghosh, A. E. Garcia, and S. Garde, "Studying pressure denaturation of a protein by molecular dynamics simulations," Proteins: Struct., Funct., Bioinf. 78, 1641-1651 (2010).

${ }^{51}$ Y. Djikaev and E. Ruckenstein, "Recent developments in the theoretical, simulational, and experimental studies of the role of water hydrogen bonding in hydrophobic phenomena," Adv. Colloid Interface Sci. 235, 23-45 (2016).

${ }^{52}$ B. Widom, P. Bhimalapuram, and K. Koga, "The hydrophobic effect," Phys. Chem. Chem. Phys. 5, 3085-3093 (2003).

${ }^{53} \mathrm{~A}$. Luzar, S. Svetina, and B. Zeks, "Consideration of the spontaneous polarization of water at the solid/liquid interface," J. Chem. Phys. 82, 5146-5154 (1985).

${ }^{54} \mathrm{M}$. G. Ortore, F. Spinozzi, P. Mariani, A. Paciaroni, L. R. Barbosa, H. Amenitsch, M. Steinhart, J. Ollivier, and D. Russo, "Combining structure and dynamics: Non-denaturing high-pressure effect on lysozyme in solution," J. R. Soc., Interface 6, S619-S634 (2009). 